\title{
CLIENT/SERVER ACCESS TO THE SIMBAD DATABASE WITHIN THE ALADIN PROJECT
}

\author{
F. BONNAREL, E. DIVETAIN, F. OCHSENBEIN, Ph. PAIllou and $M$. \\ WENGER \\ Observatoire Astronomique de Strasbourg \\ Centre de Données astronomiques de Strasbourg \\ 11 rue de l'Université \\ F-67000 Strasbourg, France
}

SUMMARY. Aladin, the Deep Sky interactive Facility, is a new CDS project which provides interactive access to both sky surveys, (starting with optical ones) and also to catalogue data about the studied field objects (Paillou et al. 1993).

A prototype of Aladin, the deep sky interactive facility at CDS, was developed in 1993. In this prototype, the user interface directly communicates with the SIMBAD database and with a set of catalogues extracted from the CDS catalogues archive, in a client/server mode.

\section{The SIMBAD Server}

This paper addresses the Aladin interface to SIMBAD, based on a client-server model. In the Aladin client/server model, the server program communicates with a client program through the network. Additionally, the server must also be able to manage the communication with several clients at the same time (e.g. to open and close connections).

\subsection{IMPLEMENTATION}

The SIMBAD software was not originally (1988) written following this approach; and to achieve compatibility with the original SIMBAD architecture, the functionality of the SIMBAD software was moved to a subroutine named simbadssp; and a server mode was added to the 'user interface class' (input/output manager facility). There are therefore two versions of the main program which can call the simbadssp subroutine: a 'classical' version, which is still used by all the 'normal' SIMBAD users; and a 'server' version, which performs the following functions:

- listening to the client connection requests;

- forking a 'child' process to execute the simbadssp subroutine;

- waiting for another client request.

The interrogation facility of SIMBAD is thus duplicated for each client. The input/output manager of the simbadssp subroutine is set to the client server mode. In this mode, SIMBAD is reading and writing on the socket provided by the server main program.

In this configuration SIMBAD and the client programs use a simple communication protocol, based on the following server states: DATA, LIST, ERROR, PROMPT, REFUSED. The PROMPT state tells the client that SIMBAD has finished sending data and is waiting for new 
commands. Between two PROMPTs the data are buffered and sent to the client. The ERROR state indicates that SIMBAD execution encountered an error. The REFUSED state indicates that the SIMBAD session didn't start. The DATA state indicates that the server is sending data. The LIST state indicates that the server will now send data for a list of objects. Additionally, depending on the client requests, functions other than SIMBAD could be run by the server program.

\subsection{EXTENSIONS UNDER DEVELOPMENT}

These developments (server main program, 'user interface class' in server mode) can also be used within the Aladin project for the Image Database Management System or the CDS multimedia server because these two database management systems have a general design similar to the SIMBAD software (based on object orientation and on a clear separation between the 'engine' and the application).

\subsection{SIMBAD CLIENT ROUTINES}

In order to write clients like the Aladin user interface easily, without any required knowledge of the SIMBAD language, a set of client routines has been developed on top of the communication layer. These routines allow us to locate objects on the basis of identifiers or coordinates and to retrieve the corresponding data defined by simple 'astrotypes', e.g. J for J2000 position, C for the classification of the object (Ochsenbein \& Dubois 1992), M. B. for the blue magnitude, 0 . UBV for UBV observations, B for bibliographical reference codes, etc. The full titles of the bibliographical references can also be retrieved with these routines.

\section{The Catalogue Server}

For more than 20 years the CDS has maintained, in addition to the SIMBAD data-base, an archive of astronomical catalogues in machine-readable form, based on a collection of over 650 catalogues. This archive has been recently reorganised and standardised (Ochsenbein 1993). Presently this large collection is accessed primarily by the anonymous ftp service (see Ochsenbein 1993). Additionally, we are currently installing a Catalogue Server which will return to a client records extracted from astronomical catalogues, according to the following two basic criteria:

1) choice of the catalogues to be scanned, according to a predefined purpose (e.g. catalogues for photometric calibration, with IR data);

2) specification of a region of the sky to be examined.

Such a server is typically aimed at an application like Aladin; a few stand-alone client programs have been developed and are readily accessible (can be copied via ftp anonymous as the file /pub/cats/cdsclient.tar on cdsarc.u-strasbg.fr):

- findgre allows one to retrieve GSC1.1 stars; this 'Pocket Guide Star Catalogue' is described in Preite-Martinez \& Ochsenbein (1993);

- findcat allows one to retrieve the existing catalogues in CDS Archive, from some keyword (e.g. author's name, word picked from title, CDS catalogue number);

- catcat - fits allows one to copy a catalogue directly in FITS format. It should be noted that the catalogue archive is made of plain ASCII files, and the transformation to FITS is 
performed by the Catalogue Server in real time.

\section{References}

Ochsenbein, F. and Dubois, P., 1992. 'Astronomy from Large Databases II', ESO Conference and Proceeding, 43, 405.

Ochsenbein, F., 1993. Proc. ESO/OAT Workshop on 'Handling and Archiving Data from Ground-based Telescopes', Trieste, April 1993.

Paillou, Ph., Bonnarel, F., Ochsenbein, F. and Crézé, M., 1993. These proceedings.

Preite-Martinez, A. and Ochsenbein, F., 1993. Proc. ESO/OAT Workshop on 'Handling and Archiving Data from Ground-based Telescopes', Trieste, April 1993. 